\title{
Superconducting condensate residing on small Fermi pockets in underdoped cuprates
}

\author{
V. M. Krasnov \\ Department of Physics, Stockholm University, AlbaNova University Center, SE-10691 Stockholm, Sweden
}

\begin{abstract}
How does Fermi surface develop in cuprates upon doping of a parent Mott insulator, does it consist of large barrels or small pockets, which of them is responsible for superconductivity and what is a role of the pseudogap? Those are actively debated questions, important for understanding of high temperature superconductivity. Here we analyze doping dependence of interlayer tunneling in cuprates. We observe that with decreasing doping the supercurrent is rapidly decreasing, but the quasiparticle resistance at high bias remains almost unchanged. This indicates that Cooper pair and quasiparticle currents originate from different parts of Brillouin zone: Cooper pairs are residing only on small pockets, which are progressively shrinking with decreasing doping, but the quasiparticle current is integrated over the full length of barrels, which are only weakly doping dependent. The expanding pseudogap areas along the barrels do not contribute to pair current. This provides direct evidence for nonsuperconducting origin of the pseudogap.
\end{abstract}

Fermi surface in metals occurs at an intersection of a conduction band with a chemical potential. Insulators do not have Fermi surface because the chemical potential lies in the band gap region. High temperature superconductivity in cuprates appears upon doping of a Mott insulator. One of the key questions is how Fermi surface develops with doping [1, 2]. According to Luttinger theorem Fermi surface area should be proportional to doping $p$. However, for cuprates the photoemission edge, seen by angular-resolved photoemission spectroscopy (ARPES), does not grow gradually with doping but forms large barrels with an area $\propto(1+p)$ already at the lowest doping [2] 4. However, strictly speaking only nodal parts of the barrels (Fermi arcs) are representing true Fermi surface because anti-nodal parts are gapped [4, 5]. The arcs size is growing linearly with doping [6] 9]. Luttinger theorem can be satisfied assuming that arcs represent parts of small Fermi pockets [4, 10, 11]. Although existence of small pockets was confirmed by quantum oscillation experiments [12 15], their position in Brillouin zone [1], connection to barrels and significance for high- $T_{c}$ superconductivity remains unclear [16].

The role of a normal state pseudogap (PG) is another related issue [17, 18. Similarities between the PG and the superconducting gap (SG) have led to an assumption of precursor superconductivity origin of the PG [19]. In this case anti-nodal PG parts of barrels should contain a major part of the superconducting condensate. However, there are also arguments in favor of competition of the two co-existing gaps [8, 16, 17, 20, 26. Discrimination between SG and PG is particularly difficult at $T<T_{c}$, when the whole barrel is gapped. So far it is not possible to conclude whether superconductivity is originating from large barrels or small Fermi pockets.

Here we analyze doping dependence of interlayer tunneling characteristics of small $\mathrm{Bi}_{2} \mathrm{Sr}_{2} \mathrm{Ca}_{1-x} \mathrm{Y}_{x} \mathrm{Cu}_{2} \mathrm{O}_{8+\delta}$ $[\mathrm{Bi}(\mathrm{Y})-2212]$ intrinsic Josephson junctions. We utilize the ability of superconducting tunnel junctions to independently probe Cooper pair and single quasiparticle (QP) currents. We observe that the high-bias QP resistance re- mains almost doping independent, implying that QP current is originating from weakly doping dependent barrels. To the contrary, the supercurrent is rapidly decreasing with decreasing doping, indicating that Cooper pairing occurs only on small Fermi pockets that progressively shrink with decreasing doping. The antinodal parts of barrels, which grow with underdoping, do not contribute to supercurrent. This directly proves that the PG is not due to Cooper pairing. We present numerical calculations that support our conclusions.

Intrinsic Josephson junctions are naturally formed in Bi-2212 single crystals [27. Atomic scale of such junctions leads to a large capacitance and a quality factor $Q \sim$ $10^{2} 28$. Current-Voltage $(I-V)$ characteristics of junctions with $Q \gg 1$ acquire a hysteresis with zero-voltage and resistive branches corresponding to Cooper pair and QP tunneling, respectively, 20,22, 29 33. which allows independent analysis of pair and QP transport.

We study $\operatorname{Bi}(Y)-2212$ single crystals from the same batch with an optimal $T_{c}(O P) \simeq 95 \mathrm{~K}$. Doping level $p$ was changed by annealing at $T=600^{\circ} \mathrm{C}$ and was estimated using an empirical expression $T_{c}(p)=T_{c}(O P)[1-$ $\left.82.6(p-0.16)^{2}\right]$ [17. It corresponds to an onset of superconductivity at $p=0.05$ at the insulator-to-metal transition and to the optimal doping (OP) at $p=0.16$ (holes per $\mathrm{Cu}$ ). Micron-size mesa structures, containing $N=7-12$ junctions were made using micro/nanofabrication techniques. Details of sample fabrication and characterization can be found in Refs. 20 22, 29.

Figure 1 summarizes temperature and doping dependencies of interlayer tunneling. Fig. 1 (a) shows $T$ dependencies of $I$ - $V$ 's for a moderately underdoped (UD) mesa $p \simeq 0.14$. At $T<T_{c}$ a sum-gap kink appears at $V=2 \Delta / e$ (per junction) followed by Ohmic, and almost $T$-independent tunnel resistance $R_{n}$. The constancy of $R_{n}$ is a fundamental consequence of electronic state conservation 22. Spectroscopic features are better analyzed using $d I / d V(V)$ characteristics, shown in Fig. 1 (b). It is seen that the sum-gap peak shifts to lower voltages and decreases in amplitude with increasing $T$ and van- 

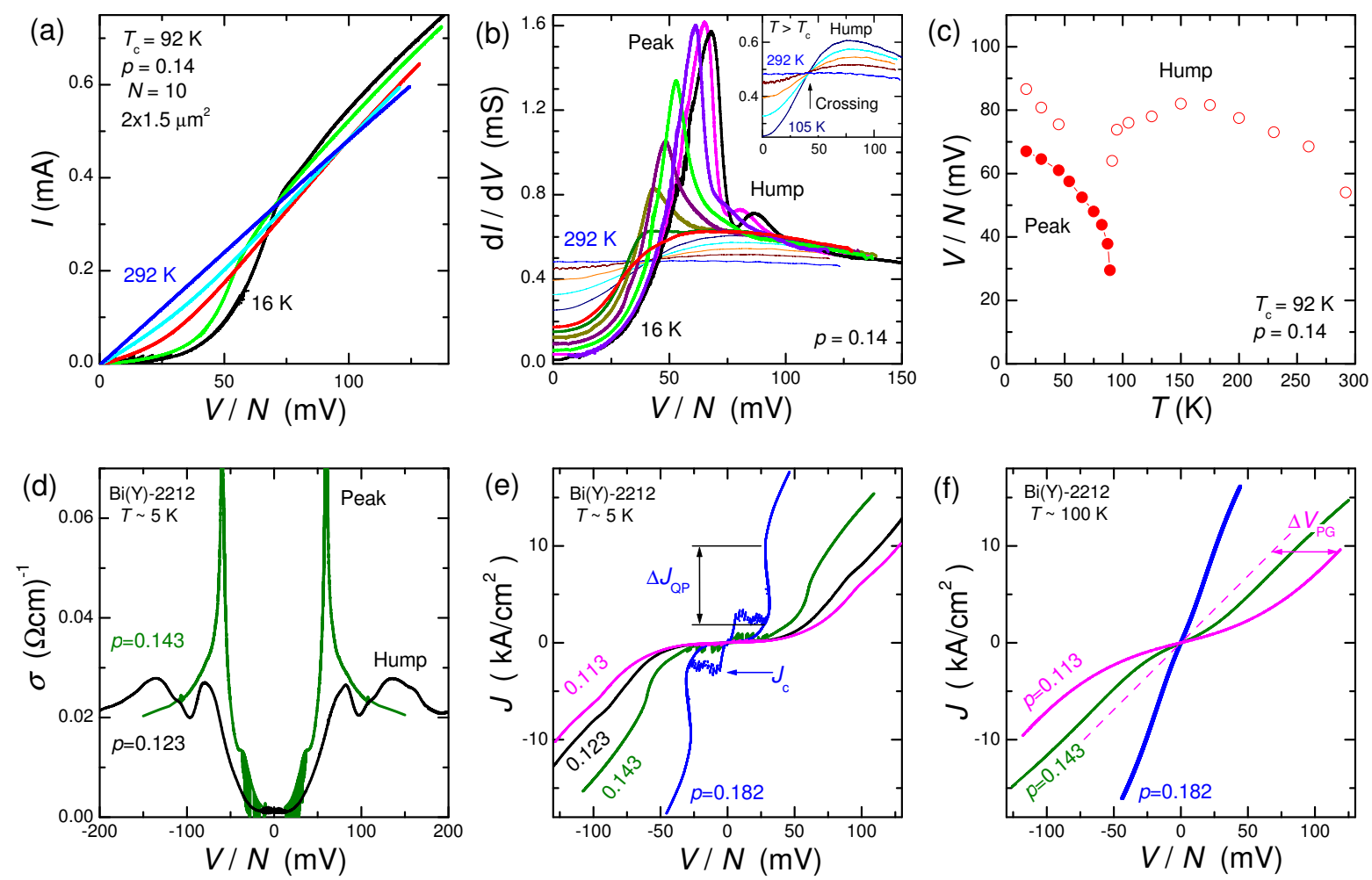

FIG. 1. (Color online) Temperature and doping dependence of intrinsic tunneling characteristics. (a) $I-V$ and (b) $d I / d V(V)$ characteristics of a small moderately underdoped mesa at different $T$. Inset demonstrates crossing of $d I / d V(V)$ curves at $T>T_{c}$. (c) Temperature dependencies of the superconducting peak and the pseudogap hump voltages for the same mesa. (d) Comparison of normalized intrinsic spectra for slightly and strongly underdopoed mesas. (e) and (f) Comparison of normalized $I-V$ curves at different doping (e) in the superconducting and (f) in the normal states.

ishes at $T_{c}$. At $T>T_{c}$ the zero-bias conductance remains suppressed as a consequence of persisting PG. The corresponding missing states are expelled into a broad hump at higher voltages. With increasing $T$ the zero bias minimum fills-in and the hump is deflated in a state conserving manner [21, 22, so that the $d I / d V(V)$ characteristics at different $T$ cross at one point, as shown in the inset 21, 29]. Fig. 1 (c) shows T-dependencies of the superconducting peak and the pseudogap hump. It is seen that the SG decreases rapidly upon approaching the $T_{c}$ in a BCS (Bardeen-Cooper-Schrieffer) manner [21]. The PG persists in a broad $T$ range both below and above $T_{c}$.

Fig. 1 (d) shows differential conductivity for a slightly $p=0.143$ and strongly $p=0.123$ UD mesas at low $T$. It is seen that for a strongly UD mesa $p=0.123$ the hump coexists with the peak at $T \ll T_{c} 20,24$, the peak is strongly suppressed, the hump is enhanced and has even larger amplitude than the peak. With increasing doping the peak height is increasing and the hump is decreasing both in height and voltage. With further increase of doping the hump is buried under the peak, as seen from Fig. 1 (d) for $p=0.143$. The hump, however, is uncovered at elevated $T$ as the peak shifts to lower voltages [20].

Fig. 1 (e) and (f) represent current density $J$ vs. volt- age per junction characteristics for different doping levels (e) at low $T$ and (f) above $T_{c}$. The following main features, which will be in focus of our discussion, are seen:

(i) The critical current. Multiple branches at low bias appear due to one-by-one switching of junctions from the superconducting to the resistive state [20, 27]. The amplitude of the branches represent the Josephson critical current density $J_{c}$, marked in Fig. 1 (e). $J_{c}$ rapidly decreases with decreasing doping. As a result, branches, which are very pronounced for the overdoped (OD) mesa $p=0.182$, are hardly visible (on this scale) for the most UD $p=0.113$ mesa. Reduction of $J_{c}$ reflects reduction of the superconducting condensate with decreasing doping.

(ii) The sum-gap kink amplitude. $\Delta J_{Q P}$ represents a number of QP states within the superconducting gap, which are subjected to pairing. Therefore, $\Delta J_{Q P}$ and $J_{c}$ should be directly connected. Indeed, from Fig. 1 (e) it is seen that $\Delta J_{Q P}$ is also rapidly decreasing with decreasing doping so that the kink becomes poorly visible for the most UD mesa. This indicates a reduction of the number of electronic states subjected to Cooper pairing.

(iii) The pseudogap is most clearly visible in the normal state when superconducting features are gone. From Fig. 11 (f) it is seen that the $I-V$ of a strongly UD mesa $p=$ 

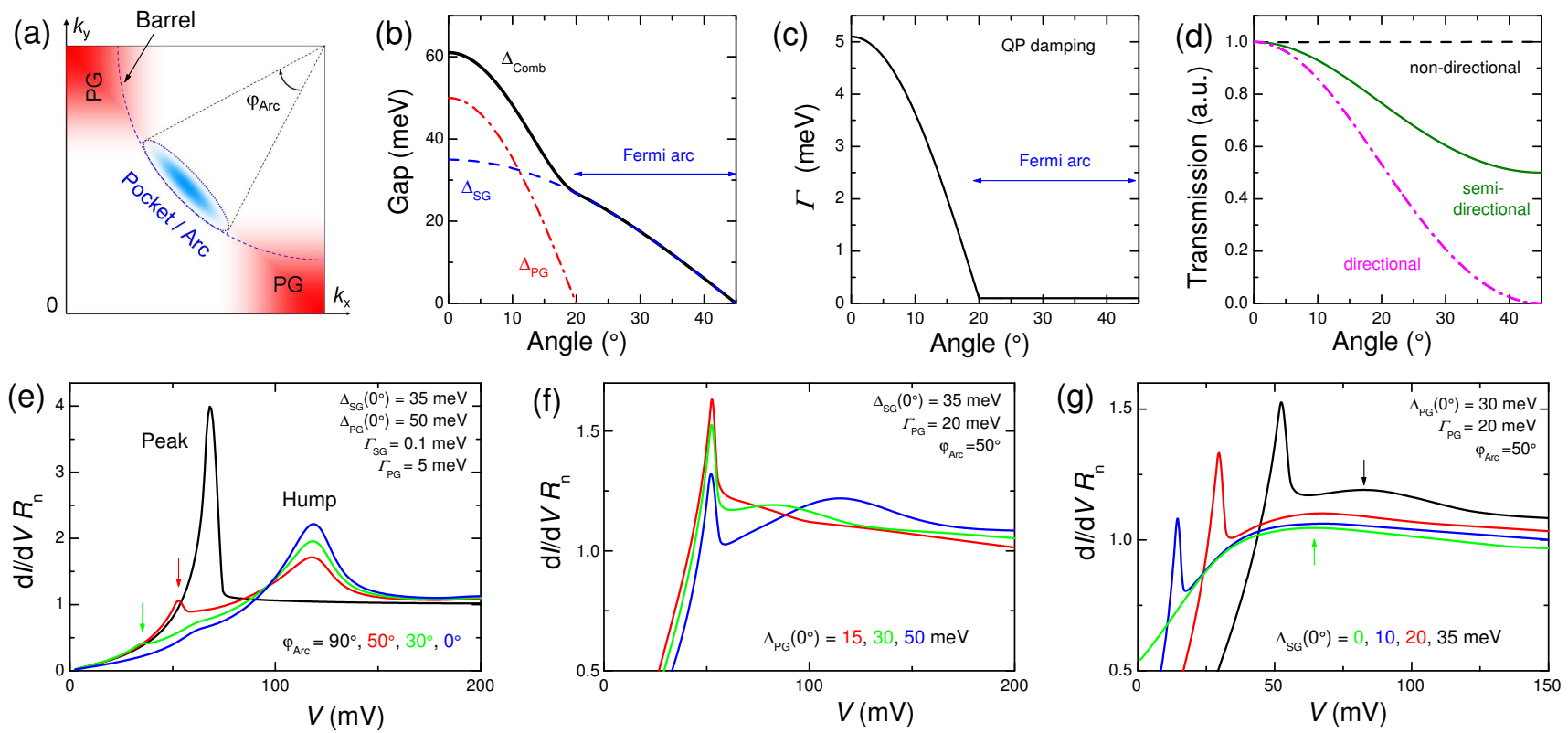

FIG. 2. (Color online) Numerical modelling of intrinsic tunneling characteristics. (a) Sketch of a quarter of the Brillouin zone. (b)-(d) Angular distribution along the barrel from an antinodal to a nodal point of (b) the gaps, (c) the quasiparticle damping factor and (c) the tunneling transmission probability. (e)-(g) Evolution of calculated $d I / d V(V)$ curves upon varying of (e) the pocket size, (f) The pseudogap energy and (g) the superconducting gap. Arrows indicate positions of peaks and humps.

0.113 is nonlinear at $T>T_{c}$. The current is suppressed below a threshold voltage $\Delta V_{P G} . \Delta V_{P G}$ decreases with increasing doping and disappears for slightly OD mesas, as seen from an almost Ohmic $I-V$ at $p=0.182$.

(iv) The tunnel resistance. From Fig. 1 (e) it is seen that the high-bias $R_{n}$ is initially increasing with decreasing doping, but for UD mesas the differential resistance becomes almost doping independent. Since tunnel resistance depends on the QP DoS it is expected that $R_{n}$ should increase with decreasing carrier concentration and saturation at low doping requires explanation.

To gain a better understanding of experimental characteristics we performed numerical analysis taking into account a symmetry of the order parameter and a topology of the Fermi surface, as shown in Fig. 2(a). Following recent studies [10 12, we assume that central parts of large barrels represent small Fermi pockets with an angular size $\varphi_{A r c}$. Fig. 2 (b) represents angular dependencies of the gaps along the barrel. In the normal state only antinodal PG parts of barrels are gapped. At $T<T_{c}$ a $\mathrm{d}$-wave $\mathrm{SG}$ is opening. We assume that the antinodal regions acquire a combined gap $\Delta_{C o m b}=\sqrt{\Delta_{P G}^{2}+\Delta_{S G}^{2}}$. The exact scenario in the antinodal region is not very important because the main difference between barrel and arc regions is in the QP damping factor $\Gamma$, which is small at the pocket and large at the barrel, as shown in Fig. 2 (c). This makes QP's ill-defined in the PG region [2]. Finally, we have to take into account angular dependence of the transmission probability $\operatorname{Tr}(\varphi)$. For non-directional tunneling $\operatorname{Tr}(\varphi)=$ const. However, $c$-axis transport in cuprates should be dominated by antinodal regions. The corresponding directional $\operatorname{Tr}(\varphi)$ is shown by the dasheddotted line in Fig. 2 (d). We have found that the experimental data is best fitted using a semi-directional $\operatorname{Tr}(\varphi)$, which is finite at nodal regions, as shown by the solid line in Fig. 2 (d). Numerical simulations presented in Figs. 2 (e-g) are made for the semi-directional case. Details of calculations can be found in the Supplementary [34].

Fig. 2 (e) demonstrates variation of $d I / d V(V)$ upon changing of the arc size. The case $\varphi_{\text {Arc }}=90^{\circ}$ corresponds to the absence of the PG. In this case the spectrum contains a single superconducting peak at $\mathrm{eV}=$ $2 \Delta_{S G}\left(0^{\circ}\right)$ with the height and the shape similar to that for OP mesas. As the arc shrinks, the amplitude of the peak is rapidly decreasing and the $\mathrm{PG}$ hump is growing at $e V=2 \Delta_{C o m b}\left(0^{\circ}\right)$. This is similar to evolution of experimental curves with decreasing doping in Fig. 1 (d).

Fig. 2 (f) demonstrates variation of $d I / d V(V)$ upon changing the PG energy. When $\Delta_{P G}$ is significantly larger than $\Delta_{S G}$ both the peak and the hump are well defined. However, as $\Delta_{P G}$ starts to decrease the hump is moving towards the peak and is eventually buried under the peak. This is similar to evolution of experimental curves with increasing doping, see Figs. 1 (b) and (d).

Fig. $2(\mathrm{~g})$ shows variation of $d I / d V(V)$ upon changing of $\Delta_{S G}$, mimicking $T$-variation shown in Fig. 1 (b). Note that the hump moves to lower voltages with decreasing $\Delta_{S G}$ because in our case it occurs at the combined gap. We observe a similar shift of the hump at $T<T_{c}$ in experiment, as shown in Fig. 1 (c). We conclude that 

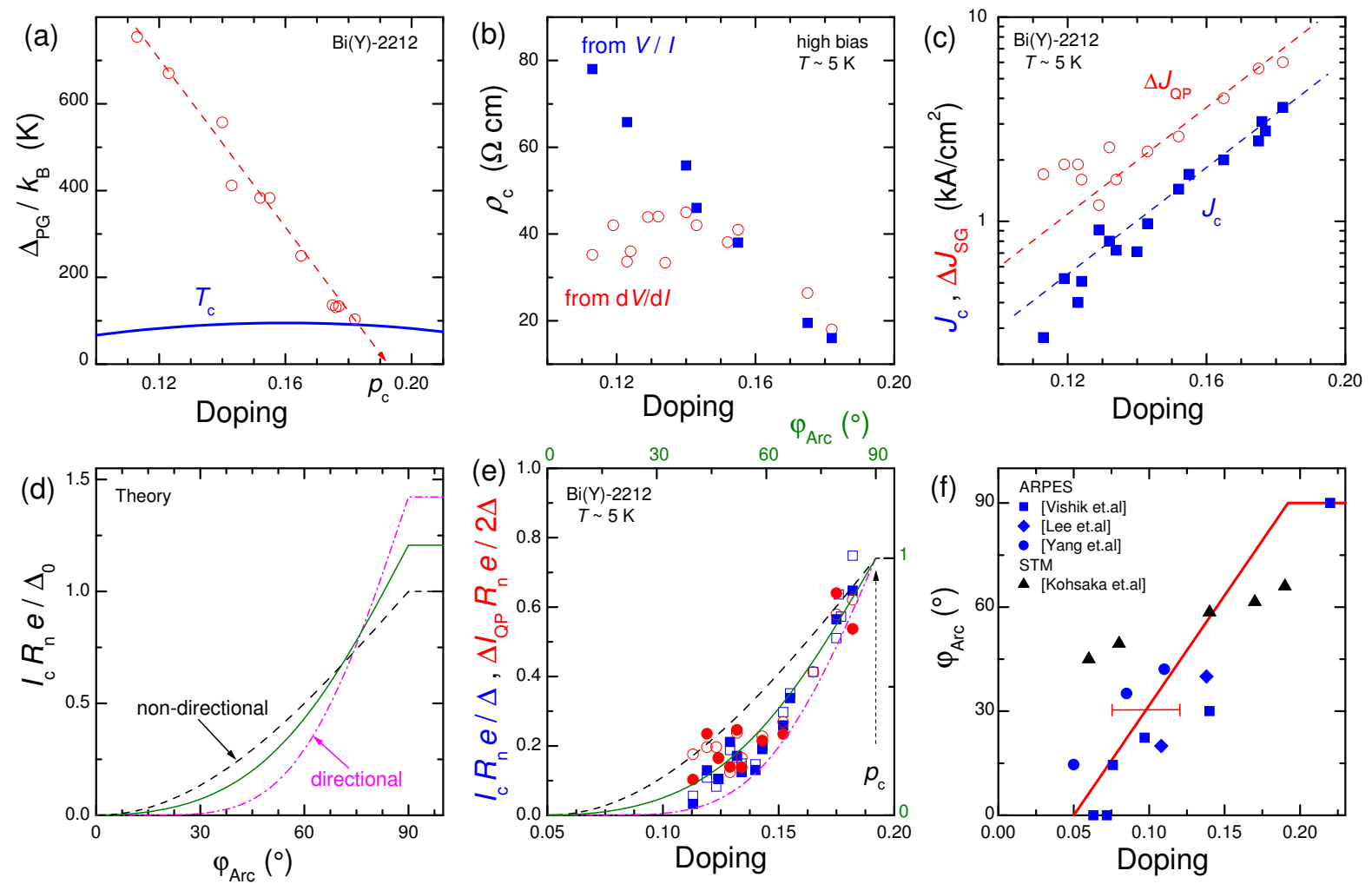

FIG. 3. (Color online) Doping dependence of (a) the pseudogap, (b) high-bias resistivities, (c) the critical current density $J_{c}$ and the amplitude of the sum-gap kink $\Delta J_{Q P}$. (d) Calculated dependence of the critical current from Fermi pockets, as a function of $\varphi_{\text {Arc }}$ for non-directional, semi-directional and directional tunneling (dashed, solid and dashed-dotted lines). (e) Comparison of scaled $I_{c}$ and $\Delta I_{Q P} / 2$. They represent the amounts of Cooper pairs and QP states subjected to pairing, respectively. Lines (top-right axes) represent normalized curves from panel (d). The reduction of $I_{c}$ and $\Delta I_{Q P}$ with decreasing doping is consistent with proportional shrinkage of the Fermi pockets containing the superconducting condensate. (f) The solid line represents a deduced doping dependence of the pocket size. Symbols represent corresponding data obtained by other techniques.

there is a good overall agreement between the experimental data and the considered model. Therefore, we employ this model for a quantitative analysis of data.

Figure 3 represents a summary of doping dependence of intrinsic tunneling characteristics. Fig. 3 (a) shows the PG energy obtained from half of the PG hump voltage. It is linearly decreasing with increasing doping and tends to vanish at the quantum critical point slightly above $p_{c}=0.19$, in agreement with Refs. 8, 17. A topological barrel-pocket transition should occur at this point [12.

In Fig. 3 (b) we analyze doping dependence of high bias resistivity. Solid and open symbols represent dc and differential (ac) values, respectively. From Fig. 1 (f) it is seen that for OD mesas the $I-V$ is Ohmic and the two resistances coincide. The $I-V$ 's of UD mesas become non-linear due to appearance of the PG and the two resistances become different. The linear doping dependence of the dc resistivity reflects the corresponding behavior of the PG, Fig. 3 (a). However, the ac resistivity becomes almost doping independent at low doping, as seen from Fig. 1 (e). Simulations provide a clarification of different behavior of dc and ac resistivities. From Figs. 2 (e) and (f) it is seen that the high-bias $d I / d V$ is independent of $\varphi_{A r c}$ and $\Delta_{P G}$. This occurs because the PG does not change the total amount of states but just redistributes them. At a sufficiently high voltage $e V \gg \Delta_{P G}$ all QP states along the barrels, including the gapped anti-nodal parts, contribute to the QP current. Therefore, the weak doping dependence of ac-resistivity is a consequence of the weak doping dependence of barrels [3, 4].

Fig. 3 (c) shows doping dependence of $J_{c}$ and $\Delta J_{Q P}$ It is seen that unlike $R_{n}$ both $J_{c}$ and $\Delta J_{Q P}$ decay rapidly with decreasing doping. Behavior of $J_{c}(p)$ is in agreement with previous reports 24, 30-32. A qualitative difference of doping dependencies of the Cooper pair and the high-bias QP transport is the main new observation of this work. As explained above, the weak doping dependence of $R_{n}$ suggests that the QP current is integrated over the full length of barrels, which are weakly doping dependent. Therefore, a rapid decrease of the Cooper pair current with decreasing doping indicates that the superconducting condensate does not reside along the full length of barrels, but occupy a progressively smaller fraction, as expected for small Fermi pockets. 
To understand how $I_{c}$ depends on the pocket size we performed corresponding calculations, shown in Fig. 3 (d) (see Ref. 34]). In the non-directional case,

$$
I_{c}=\frac{\Delta_{0}}{e R_{n}}\left[1-\cos \left(\varphi_{A r c}\right)\right] .
$$

In the absence of the $\mathrm{PG}, \varphi_{A r c}=90^{\circ}, I_{c} R_{n}=\Delta_{0} / e$. With decreasing doping Fermi arcs shrink and $I_{c}$ decreases. The $I_{c}$ vanishes when arcs collapse $\varphi_{\text {Arc }} \rightarrow 0$. The supercurrent and the high-bias QP resistance have different doping dependencies because supercurrent is measured at zero voltage. Consequently, pair tunneling occurs only between true (ungapped) Fermi surfaces, i.e., Fermi pockets, which shrink with decreasing doping. To the contrary, QP tunneling occurs at finite bias and at $e V \gg \Delta_{P G}$ it collects all QP states along the barrels.

From Fig. 3 (c) it is seen that $\Delta I_{Q P}$ and $I_{c}$ are changing in a correlated manner. For a d-wave tunneling in the absence of the PG the kink amplitude should be twice the critical current $\Delta I_{Q P}=2 \Delta_{0} / e R_{n}=2 I_{c}$. In Fig. 3 (e) we show the correspondingly scaled quantities as a function of doping. They were obtained using the high bias differential resistance as $R_{n}$ (open and solid symbols were obtained using different criteria for estimation of $R_{n}$ and $\Delta)$. It is seen that $I_{c}$ and $\Delta I_{Q P} / 2$ merge together, confirming that both are indeed related: $I_{c}$ is measuring the amount of Cooper pairs and $\Delta I_{Q P}$ the amount of QP states within the gap, subjected to pairing. Importantly, the scaling indicates that both quantities originate from the same parts of the Brillouin zone ( i.e., Fermi pockets). The reduction of both $I_{c}$ and $\Delta I_{Q P}$ with decreasing doping reflects the shrinkage of the pocket size.

In order to estimate variation of $\varphi_{\text {Arc }}$ with doping, in Fig. 3 (e) we compare experimental data with theoretical curves from Fig. 3 (d). Here we assumed that $\varphi_{\text {Arc }}=90^{\circ}$ at the onset of the $\mathrm{PG}, p=0.192$, and that $\varphi_{\text {Arc }}=$ $0^{\circ}$ at the insulator-to-metal transition, $p=0.05$. The agreement is remarkable taking into account that there is no fitting other than adjustment of the vertical scale.

Figure 3 (f) summarizes our main result. A solid line represents the deduced angular size of the Fermi pocket/arc as a function of doping. It shrinks linearly with decreasing doping, consistent with previous reports by other techniques [6, 8, 9, 11, shown for comparison in the figure. An important new aspect of our work is that we could discriminate between Cooper pair and quasiparticle transport. We conclude that the QP current at high bias is originating from the full area of large barrels, leading to weak doping dependence of $R_{n}$. However, the Cooper pair current originates only from small Fermi pockets, which shrink with decreasing doping, leading to a rapid decrease of both the critical current and the sum-gap kink amplitude. The pseudogap parts of barrels, which grow bigger with underdoping, apparently do not contribute to supercurrent. This is a direct evidence for non-superconducting origin of the pseudogap in cuprates.
Technical support from the Core Facility in Nanotechnology at SU is gratefully acknowledged.

\section{SUPPLEMENTARY INFORMATION}

The supplementary material provides additional information about numerical calculations of interlayer tunneling characteristics and analytical results for variation of $I_{c} R_{n}$ as a function of the arc size for non-directional, directional and semi-directional energy and momentum conserving tunneling between d-wave superconductors. We assumed that the supercurrent is originating only from nodal arcs and single quasiparticle current from the full length of large barrels.

\section{Calculation of interlayer tunneling characteristics}

Proper calculation of interlayer tunneling characteristics requires accurate integration of tunneling current over all initial and final states, taking into account the band structure, angular dependence of the energy gap $\Delta(\varphi)$ and the transmission probability $\operatorname{Tr}\left(\varphi_{1}, \varphi_{2}\right)$ for tunneling between the initial and final states with the momentum angles $\varphi_{1,2}$, where $\varphi$ is the angle between the momentum of the QP and the principle axis of the Brillouin zone. We consider the case of elastic tunneling, in which the energy of electrons is conserved. In this case the QP tunneling current between two adjacent superconducting layers can be written as:

$$
I=A \int_{0}^{2 \pi} \frac{d \varphi_{1}}{2 \pi} \int_{0}^{2 \pi} \frac{d \varphi_{2}}{2 \pi} \int_{-\infty}^{+\infty} d E
$$

$\operatorname{Tr}^{2}\left(\varphi_{1}, \varphi_{2}\right) N\left(E, \varphi_{1}\right) N\left(E+e V, \varphi_{2}\right)[f(E)-f(E+e V)]$,

where $E$ is the energy of the QP with respect to the chemical potential, $N\left(E, \varphi_{1}\right)$ and $N\left(E+e V, \varphi_{2}\right)$ are the corresponding QP DoS in the initial and final states:

$$
N(E, \varphi)=N(0) \Re\left[\frac{E-i \Gamma(\varphi)}{\sqrt{(E-i \Gamma(\varphi))^{2}-\Delta(\varphi)^{2}}}\right] .
$$

Here $\Gamma(\varphi)$ is the angular dependent quasiparticle damping factor (the inverse QP lifetime). In the absence of the PG, analysis of various tunneling scenarios for coherent $\varphi_{1}=\varphi_{2}$ and incoherent $\varphi_{1} \neq \varphi_{2}$ tunneling between $\mathrm{d}$-wave superconductors can be found in the supplementary material to Ref. [29] and in Ref. [35]. Here we will focus on analysis of the pseudogap effect for the case of coherent $\varphi_{1}=\varphi_{2}$ and elastic (momentum and energy conserving) tunneling for a model of a "remnant" Fermi barrel with a PG in antinodal and a Fermi-arc in the nodal parts, as shown in Fig. 2 (a). We assume that Cooper pair tunneling occurs only in the Fermi-arc regions, but the QP tunneling occurs along the whole barrel. All the characteristics are presented for a quarter of 
Brulloin zone and are symmetrically reflected for other three quarters of the zone.

Fig. 2 (b) represents angular dependence of the gaps. In the normal state only PG is present in the antinodal parts of the barrels in the angular interval from 0 to $\varphi_{P G}=\pi / 4-\varphi_{A r c} / 2$. We assumed the following angular dependence,

$$
\Delta_{P G}(\varphi)=\Delta_{P G}(0) \cos \left(\frac{\pi \varphi}{2 \varphi_{P G}}\right)
$$

shown by the dashed-dotted line in Fig. 2 (b).

At $T<T_{c}$ there is a superconducting gap with a dwave symmetry,

$$
\Delta_{S G}(\varphi)=\Delta_{0} \cos (2 \varphi)
$$

shown by the dashed line in Fig. 2 (b). We assume that in the PG region the two gaps form a combined gap

$$
\Delta_{C o m b}(\varphi)=\sqrt{\Delta_{P G}^{2}(\varphi)+\Delta_{S G}^{2}(\varphi)}
$$

shown by the solid line in Fig. 2 (b). This assumption is, however, not critical because antinodal parts of the barrel do not contribute to a sharp sum-gap peak in $d I / d V$ because of a large QP damping in the PG region [2, as shown in Fig. 2 (c). We assumed that within the PG region the $\Gamma_{P G}$ is varying in a similar manner to Eq. (4) with the maximum $\Gamma_{P G}(0) \sim 10 \mathrm{meV}$. In the arc region the QP damping is two orders of magnitude smaller $\Gamma_{S G}=0.1 \mathrm{meV}$. Due to the large difference in $\Gamma$ sharp sum-gap kink/peak features in $I-V$ and $d I / d V$ originate solely from the arc region and correspond to the superconducting gap. Eq. (6) simply represents a comfortable way to connect the PG and SG regions by a continuous line.

For the angular dependence of the transmission probability $\operatorname{Tr}(\varphi)$ we considered three scenarios:

(i) Non-directional tunneling $\operatorname{Tr}(\varphi)=$ const, as shown by the dashed line in Fig. 2 (d).

(ii) Directional tunneling with maximum in antinodal and zero transmission in nodal regions 36], $\operatorname{Tr}(\varphi) \propto$ $\left[\cos \left(k_{x}\right)-\cos \left(k_{y}\right)\right]^{2} \propto[\cos (\pi \sin (\varphi))-\cos (\pi \cos (\varphi))]^{2}$, as shown by the dashed-dotted line in Fig. 2 (d). This expression is well approximated by the function $\cos (2 \varphi)^{2}$, which we will use for analytical calculations below.

(iii) Semi-directional tunneling, which is an average of non-directional and directional cases, $\operatorname{Tr}(\varphi) \propto 1+$ $[\cos (\pi \sin (\varphi))-\cos (\pi \cos (\varphi))]^{2} / 4$, as shown by the solid line in Fig. 2 (d).

Finally, the QP density of states $N(\varphi)$ is likely to vary along the barrel. However, in the absence of a confident knowledge of the angular dependence of DoS we just assumed it to be constant $N(\varphi)=N(0)$. The angular variation of DoS would have the same affect as angular variation of the transmission coefficient.
Figure 4 shows calculated $I-V$ characteristics in case of semi-directional tunneling, corresponding to $d I / d V$ curves in Fig. 2 (e-g). Figs. 4 (a-c) demonstrates variation of $I-V$ 's upon changing (a) the arc size, (b) the PG energy and (c) $\Delta_{S G}$, while keeping other parameters constant. Note that the high bias resistance remains the same, irrespective of the PG. This occurs because in our case the PG is a state-conserving gap, see Eq. (3). Therefore the same current is recovered upon integration over the full barrel at high enough bias, irrespective whether there is a PG or not. Note that curves in Figs. 4 (b) and (c) calculated for a large $\Gamma_{P G}(0)=20 \mathrm{meV}$ resemble the experimental characteristics for UD mesas with a finite offset voltage $\Delta V_{P G}$, see Figs. 1 (e) and (d).

\section{Dependence of the critical current on the arc size}

For a junction made of s-wave superconductors $I_{c} R_{n}$ is independent of the transmission coefficient and is given by Ambegaokar-Baratoff expression,

$$
I_{c} R_{n}\left(T \ll T_{c}\right)=\frac{\pi \Delta}{2 e} .
$$

For coherent tunneling in a junction made of d-wave superconductors, Eq. (7) is still valid for a specific angle [37, i.e. for $\delta I_{c}(\varphi) \delta R_{n}(\varphi)$, where $\delta I_{c}(\varphi)$ and $\delta R_{n}(\varphi)$ are contributions to the critical current and resistance from Cooper pairs and QP's with momentum in the direction $\varphi$. Thus, $\delta I_{c}(\varphi)=\pi \Delta(\varphi) / 2 e \delta R_{n}(\varphi)$. From Eq. (2) $1 / \delta R_{n}(\varphi)=A e N(0)^{2} \operatorname{Tr}(\varphi)^{2} \delta \varphi / 2 \pi$. Introducing a quantity $R_{1}=A e N(0)^{2}$ for resistance at unit transmission $\operatorname{Tr}=1$, we can write expressions for the total critical current and the normal resistance, taking into account dwave angular dependence of the gap $\Delta(\varphi)=\Delta_{0} \cos (2 \varphi)$ :

$$
\begin{gathered}
I_{c} R_{1}=\frac{4}{\pi} \int_{\pi / 4-\varphi_{A r c} / 2}^{\pi / 4} \operatorname{Tr}(\varphi)^{2} \cos (2 \varphi) d \varphi \\
R_{1} R_{n}^{-1}=\frac{4}{\pi} \int_{0}^{\pi / 4} \operatorname{Tr}(\varphi)^{2} d \varphi
\end{gathered}
$$

Here we assumed that Cooper pair current is originating only from the arc and single QP current is accumulated over the full length of the barrel.

After integration of Eqs. (8) and (9) we obtain :

$$
I_{c} R_{n}=\frac{\Delta_{0}}{e}\left[1-\cos \left(\varphi_{A r c}\right)\right]
$$

for the nondirectional case $\operatorname{Tr}(\varphi) \propto 1$. For $\varphi_{\text {Arc }}=\pi / 2$ it reduces to a known expression $I_{c} R_{n}=\Delta_{0} / e$ [37].

$$
\begin{gathered}
I_{c} R_{n}=\frac{\Delta_{0}}{e} \frac{8}{3} * \\
{\left[\frac{8}{15}-\cos \left(\varphi_{A r c}\right)+\frac{2}{3} \cos \left(\varphi_{A r c}\right)^{3}-\frac{1}{5} \cos \left(\varphi_{A r c}\right)^{5}\right]}
\end{gathered}
$$



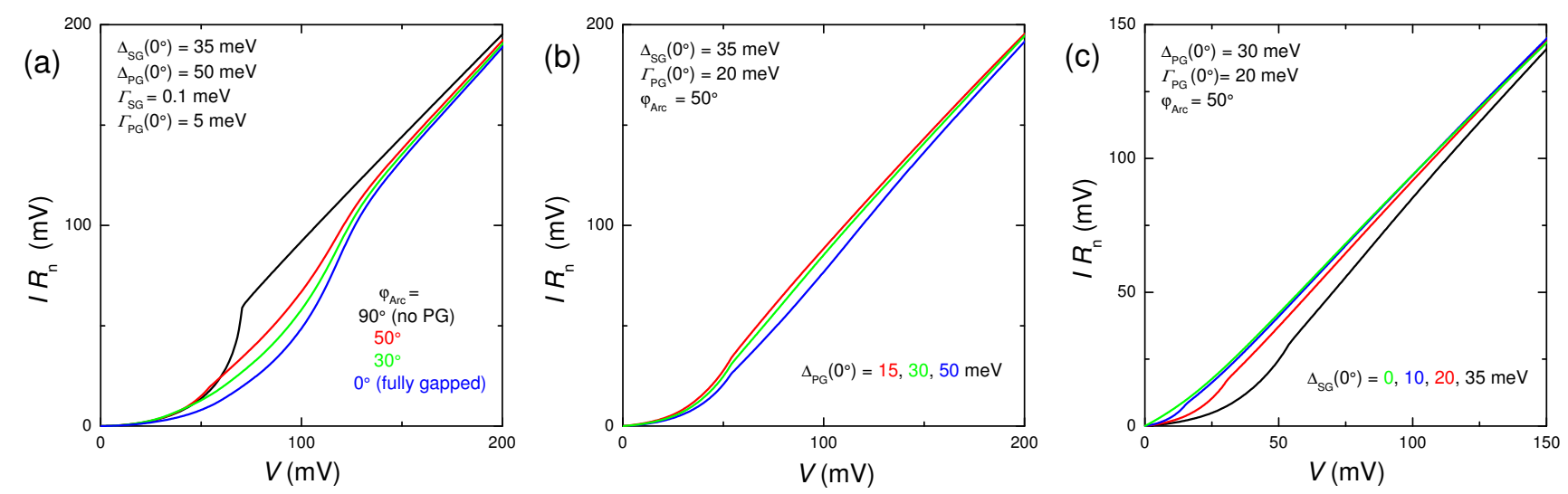

FIG. 4. Numerical modelling of intrinsic tunneling characteristics. Evolution of calculated $I$ - $V$ curves upon varying of (a) the pocket size, (b) The pseudogap energy and (c) the superconducting gap. Note that the high-bias resistance remains unchanged because it is integrated over the same barrel.

for the directional case, $\operatorname{Tr}(\varphi) \propto \cos (2 \varphi)^{2}$, and

$$
\begin{gathered}
I_{c} R_{n}=\frac{\Delta_{0}}{e} \frac{8}{19} * \\
{\left[\frac{43}{15}-4 \cos \left(\varphi_{A r c}\right)+\frac{4}{3} \cos \left(\varphi_{A r c}\right)^{3}-\frac{1}{5} \cos \left(\varphi_{A r c}\right)^{5}\right],}
\end{gathered}
$$

for the semi-directional case, $\operatorname{Tr}(\varphi) \propto 1+\cos (2 \varphi)^{2}$. The corresponding curves $I_{c} R_{n}\left(\varphi_{A r c}\right)$ are shown in Fig. 3 (d). In all cases $I_{c}$ vanishes as arcs collapse $\varphi_{A r c} \rightarrow 0$ because we assumed that the supercurrent is originating only from arc regions. The $R_{n}$ is independent of $\varphi_{\text {Arc }}$ because the QP current is integrated over the whole barrel, which we assumed to be unchanged. As described in the manuscript, such model provides a qualitative explanation of different doping dependencies of the critical current density and the high-bias differential resistance.

* E-mail: Vladimir.Krasnov@fysik.su.se

[1] W. E. Pickett, H. Krakauer, R.E. Cohen, and D.J. Singh, Science 255, 46 (1992).

[2] A. Damascelli, Z. Hussain, Z.-X. Shen, Rev. Mod. Phys. 75, 473 (2003).

[3] A. A. Kordyuk et al., Phys. Rev. B 66, 014502 (2002).

[4] F. Ronning et al., Phys. Rev. B 67, 165101 (2003).

[5] A. Kanigel et al., Nature Phys. 2, 447 (2006).

[6] W. S. Lee et al., Nature 450, 81 (2007).

[7] S.-I. Ideta et.al, Phys. Rev. B 85, 104515 (2012).

[8] I. M. Vishik et.al, PNAS 109, 18332-18337 (2012).

[9] Y. Kohsaka, et.al, Nature 454, 1072-1078 (2008).

[10] J. Meng et al., Nature 462, 335-338, (2008).

[11] H.-B. Yang et.al., Phys. Rev. Lett. 107, 047003 (2011).

[12] B. Vignolle et.al., C. R. Physique 12, 446460 (2011).

[13] E.A. Yelland et al., Phys. Rev. Lett. 100, 047003 (2008).
[14] S.C. Riggs et.al., Nature Phys. 7, 332-335 (2011).

[15] N. Barisic et.al., Nature Phys. 9, 761-764 (2013).

[16] S. Chakravarty, Rep. Prog. Phys. 74, 022501 (2011).

[17] J.L. Tallon and J.W. Loram, Physica C 349, 53 (2001).

[18] M.R, Norman, D.Pines, and C. Kallin, Adv. Phys. 54, 715-733 (2005).

[19] H.-B. Yang, J. D. Rameau, P. D. Johnson, T. Valla, A. Tsvelik, and G. D. Gu, Nature 465, 77-80 (2008).

[20] V.M. Krasnov, A. Yurgens, D. Winkler, P. Delsing and T. Claeson, Phys. Rev. Lett. 84, 5860 (2000).

[21] V. M. Krasnov, Phys. Rev. B 79, 214510 (2009).

[22] V. M. Krasnov, H. Motzkau, T. Golod, A. Rydh, S. O. Katterwe and A. B. Kulakov Phys. Rev. B 84, 054516 (2011).

[23] Th. Jacobs et.al., Phys. Rev. B 86, 214506 (2012).

[24] V. M. Krasnov, Phys. Rev. B 65, 140504(R) (2002).

[25] E. Gull, O. Parcollet, and A.J. Millis, Phys. Rev. Lett. 110, 216405 (2013).

[26] T.Ekino, A.M.Gabovich, M.S.Li, M.Pekala, H.Szymczak, and A.I.Voitenko, J. Phys. Cond. Mat. 20, 425218 (2008).

[27] R. Kleiner and P. Müller, Phys. Rev. B 491327 (1994).

[28] S. O. Katterwe, A.Rydh, H. Motzkau, A.B. Kulakov, and V. M. Krasnov Phys. Rev. B 82, 024517 (2010).

[29] S.O. Katterwe, A.Rydh, and V. M. Krasnov, Phys. Rev. Lett. 101, 087003 (2008).

[30] A. Irie and G.-I. Oya, Physica C 367 393-399 (2002).

[31] K. Inomata, T. Kawae, K. Nakajima, S.-J. Kim, and T. Yamashita, Appl. Phys. Lett. 82769 (2003).

[32] H. Kambara, I. Kakeya, and M. Suzuki, Phys. Rev. B 87 214521 (2013).

[33] J.K. Ren et.al., Sci. Rep. 2, 248 (2012).

[34] See supplementary material for details of numerical calculations and for $I_{c}\left(\varphi_{\text {Arc }}\right)$ expressions.

[35] Y.Yamada and M.Suzuki, Phys. Rev. B 66, 132507 (2002).

[36] L.B. Ioffe, and A.J. Millis, Phys. Rev. B 58, 11631 (1998).

[37] Y. Tanaka, and S. Kashiwaya, Phys. Rev. B 56, 892 (1997). 